\title{
Synthesis and characterization of new injectable and degradable dextran-based hydrogels
}

\author{
João Maia ${ }^{\mathrm{a}, *}$, Lino Ferreira ${ }^{\mathrm{b}}$, Rui Carvalho ${ }^{\mathrm{c}}$, Manuel A. Ramos ${ }^{\mathrm{d}}$, Maria H. Gil ${ }^{\mathrm{a}}$ \\ ${ }^{a}$ Departamento de Engenharia Química, Universidade de Coimbra, Pinhal de Marrocos, 3030 Coimbra, Portugal \\ ${ }^{\mathrm{b}}$ INEB-Instituto de Engenharia Biomédica, Laboratório de Biomateriais, Rua do Campo Alegre 823, 4150-180 Porto, Portugal \\ ${ }^{\mathrm{c}}$ Departamento de Bioquímica, Universidade de Coimbra, Apartado 3126, 3000 Coimbra, Portugal \\ ${ }^{\mathrm{d}}$ Instituto Superior de Engenharia, Inst. Politécnico de Coimbra, 3000 Coimbra, Portugal
}

Received 19 March 2005; received in revised form 21 July 2005; accepted 27 July 2005

Available online 19 August 2005

\begin{abstract}
Injectable and degradable hydrogels are very interesting networks for drug delivery and cell transplantation applications since they can be administered in the human body in a minimally invasive way. In most cases, the crosslinking reaction occurs by photopolymerisation or free radical polymerisation; however, the use of chemical initiators may promote cell death. In the current work, injectable and degradable dextran-based hydrogels were prepared without the use of initiators. Dextran, a natural glucose-containing polysaccharide, was oxidized with sodium periodate (dexOx) and the derivatives characterized by NMR and FTIR spectroscopy's as well as by colorimetric techniques. The oxidized derivatives were crosslinked with adipic acid dihydrazide (AAD), forming a gel within 2-4 min. The obtained hydrogels were characterized by their mechanical properties, swelling and degradation behavior under physiologic conditions. In addition, the hydrogel interior morphology as well as porous structure was evaluated by scanning electron microscopy (SEM) and mercury intrusion porosimetry (MIP). MIP analysis showed that dexOx hydrogels crosslinked with $10 \%$ of AAD were macroporous with pore sizes ranging from 0.32 to $0.08 \mu \mathrm{m}$. As expected, the average pore size increased during hydrogel degradation as confirmed by SEM and MIP studies.
\end{abstract}

(C) 2005 Elsevier Ltd. All rights reserved.

Keywords: Hydrogels; Degradation; Pore size

\section{Introduction}

The hydrophilicity of polysaccharides, along with the ease in which they can be formed into hydrogels, makes these materials ideal for many biomedical applications including systems for peptide and protein delivery and scaffolds for tissue engineering applications [1-5]. The variety of saccharide monomers (ca. 200) and the variety of possible $O$-glycoside linkages result in a diversity of polysaccharide structures, which is important in the design of hydrogels with different physicochemical properties [6]. Because polysaccharides are natural products, they are usually biocompatible and some of them are biodegradable in the human body.

\footnotetext{
* Corresponding author. Tel.: +351 239798743; fax: +351 239798703.

E-mail address: joao.rm.silva@networkcontacto.com (J. Maia).
}

0032-3861/\$ - see front matter (C) 2005 Elsevier Ltd. All rights reserved. doi:10.1016/j.polymer.2005.07.089
From the polysaccharides found in nature, dextran has particular relevance in the biomedical field. For several years, dextran has been used as a macromolecular pro-drug and as a plasma expander [7]. These glucose-based polymers are available in a wide range of molecular weights and contain a high density of hydroxyl groups that make the polymers highly hydrophilic and capable of being further functionalized chemically. Dextran is also biocompatible [3] and can be degraded through the action of dextranases in various organs in the human body, including liver, spleen, kidney, and colon [7].

A common way of making hydrogels with polysaccharides involves the derivatization of the natural polymer with vinyl compounds followed by either radical $[1,2]$ or UV [8] polymerisation of the polymer derivatives. However, this approach has some limitations since it requires the use of chemicals or photo initiators, which may promote cell death $[3,9,10]$. This is an important issue when these networks are used as subcutaneous drug delivery systems and scaffolds for tissue engineering. Recently, a novel methodology has been 
proposed elsewhere [11], which do not require the use of initiators. In that case, the guluronate content of alginates was isolated and partially oxidized and subsequently cross-linked with AAD. Additionally, it was shown that the hydrogels were able to support the adhesion and growth of primary rat calvarial osteoblast cells [12]. However, this methodology requires several steps to isolate the guluronate (which is eliminated in the human body in opposition to alginate [13]), which may reduce its general application in the biomedical field. Furthermore, no insights were given about the porous structure before and during hydrogel degradation at physiologic conditions and this is of crucial importance for the use of these networks in biomedical field. This parameter is a critical factor to control swelling [14], drug release behavior [15], biological interaction inside the body [16], as well as the diffusion of nutrients to nourish cells, when encapsulated inside of the hydrogel network [17].

In this work, we describe a methodology to prepare injectable and degradable hydrogels from oxidized dextran (dexOx) and adipic acid dihydrazide (AAD) without the use of any chemical initiator. DexOx macromonomers form almost instantaneously hydrogels in the presence of AAD, a non-toxic and non-mutagenic [18] covalent crosslinking molecule. The networks were evaluated regarding their swelling and rheological properties, as well their degradation at physiologic $\mathrm{pH}$. In this last case, MIP and SEM were used to evaluate the porous structure of the networks. The results show that it is feasible to obtain dextran-based hydrogels with different degradation profiles, which may have particular relevance in the design of new drug delivery systems and scaffolds for tissue engineering.

\section{Materials and methods}

\subsection{Materials}

Dextran (from Leuconostoc mesenteroides; $M_{\mathrm{w}}=73$, $200 \mathrm{Da}$, according to the manufacturer's specification), adipic acid dihydrazide (AAD), trinitrobenzenosulfonic acid (TNBS), phosphate buffered saline (PBS), and sodium $m$-periodate were purchased from Sigma (Sintra, Portugal). Diethyleneglycol and tert-butylcarbazate (tBC) were obtained from Aldrich (Sintra, Portugal). Trichloroacetic acid was obtained from Merck (Darmstadt, Germany). Dialysis tubes with a MWCO of $12-14,000 \mathrm{Da}$ were purchased from Medicell International Ltd (London, UK). All the other chemicals and solvents used in this work were of the highest purity commercially available.

\subsection{Nuclear magnetic resonance (NMR), Fourier transform infrared (FTIR) and size exclusion chromatography (SEC) analysis}

\footnotetext{
${ }^{1} \mathrm{H}$ and ${ }^{13} \mathrm{C}$ NMR spectra were acquired on a Varian Unity 500 NMR spectrometer (Palo Alto, CA) using a 5 mm
}

broadband NMR probe. ${ }^{1} \mathrm{H}$ NMR spectra were recorded in $\mathrm{D}_{2} \mathrm{O}(60-100 \mathrm{mg}$ in $0.7 \mathrm{~mL}$; $\mathrm{pD}$ of ca. 5.0$)$ using a $90^{\circ}$ pulse and a relaxation delay of $30 \mathrm{~s}$. The water signal, used as reference line, was set at $\delta 4.75 \mathrm{ppm}$ and was partially suppressed by irradiation during the relaxation delay. A total of 16 scans were added for each ${ }^{1} \mathrm{H}$ NMR spectra. Bidimensional ${ }^{1} \mathrm{H}-{ }^{13} \mathrm{C}$ heteronuclear multiple quantum coherence spectra (HMQC) were collected as a $1024 \times$ 512 matrix covering sweep widths of 2500 and $11,500 \mathrm{~Hz}$ in the first and second dimensions, respectively, and using 64 scans/increments. Before Fourier transformation, the matrix was zero-filled to $2048 \times 2048$ and standard Gaussian weighting functions were applied in both dimensions.

FTIR spectra were recorded with a Nicolet Magna-IR 550 spectrometer (Madison, WI, USA). The dry samples were powdered, mixed with $\mathrm{KBr}$, and pressed into pellets manually. The FTIR spectra were obtained by recording 128 scans between 4000 and $400 \mathrm{~cm}^{-1}$ with a resolution of $4 \mathrm{~cm}^{-1}$.

Size exclusion chromatography was performed in a Agilent Technologies 1100 series HPLC system, equipped with a column (TSK G4000PWxl Tosoh Bioscience) and a refraction index detector (Optilab rEX) from Wyatt Technology Corporation. The injection and column system were kept at room temperature. The eluent was mili-Q water with $0.1 \mathrm{M} \mathrm{NaNO}_{3}$ at a flow rate of $0.2 \mathrm{~mL} / \mathrm{min}$. The SEC chromatograms were obtained from samples dissolved in the eluent with a concentration of $0.5 \%(\mathrm{w} / \mathrm{v})$. Calibration was made with dextran standards (Fluka Chemie AG, Switzerland) in the molecular range of $12-80 \mathrm{kDa}$.

\subsection{Synthesis of oxidized dextrans}

An aqueous solution of dextran ( $1 \mathrm{~g} ; 0.125 \%$, w/v) was oxidized with $2 \mathrm{~mL}$ of sodium periodate solution with concentrations ranging from 0.15 to $3.1 \mathrm{M}$ (to yield theoretical oxidations from 5 to $100 \%$ ), at room temperature. An equimolar amount of diethyleneglycol was added after $20 \mathrm{~h}$ to stop the oxidation reaction. The resulting solution was dialysed for 3 days against water, using a dialysis tube with a MWCO 12-14,000 Da, and then lyophilised (Labconco Corp., Kansas City, MO, USA). The isolated yields were ca. $75 \%$. The scale-up of the reaction was done using the same procedure albeit using $30 \mathrm{~g}$ of dextran and a calculated amount of periodate to yield a theoretical oxidation of $25 \%$.

\subsection{Determination of degree of oxidation (DO)}

The DO of dexOx is defined as the number of oxidized residues per 100 glucose residues (DO refers to the experimental value unless otherwise stated) and quantified by using tBC $[11,19]$. The carbazates react with aldehyde groups to form carbazones in the same way hydrazones are formed in the presence of hydrazides. After reacting an excess amount of $\mathrm{tBC}$ with the dexOx, the unreacted $\mathrm{tBC}$ 
was quantified by adding TNBS and measuring spectrophotometrically the resulting complex (trinitrophenyl derivative) at $334 \mathrm{~nm}$. Typically, solutions of dexOx $(0.5 \mathrm{~mL}, 0.001 \% \mathrm{w} / \mathrm{v})$ and $\mathrm{tBC}(0.5 \mathrm{~mL}, 0.03 \mathrm{M})$ in aqueous trichloroacetic acid (1\%) were mixed, and allowed to react for $24 \mathrm{~h}$ at room temperature. A volume of $200 \mu \mathrm{L}$ was transferred to a vial containing aqueous TNBS solution

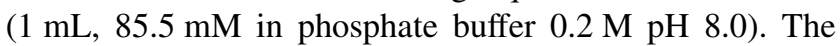
mixture was allowed to react for $60 \mathrm{~min}$ at room temperature, diluted with aqueous hydrochloric acid $(0.5 \mathrm{M})$ (the acidification results in stabilization of the coloured TNBS derivatives [20]), and the absorbance of the solution was measured at $334 \mathrm{~nm}$. Aqueous tBC solutions were used as standards to obtain a calibration curve for determining the unreacted carbazates in experimental samples. The blank used contained 1:1 double-distilled water and aqueous trichloroacetic acid (1\%, w/v).

\subsection{Formic acid titration}

In the end of the oxidation reaction (ca. $20 \mathrm{~h}$ of reaction, before the addition of diethyleneglycol), $5 \mathrm{~mL}$ of the reaction mixture were taken and transferred to a scintillation vial. The formic acid in the sample was titrated with $0.01 \mathrm{M}$ $\mathrm{NaOH}$ using phenolphthalein solution as indicator.

The oxidation reaction was also followed by ${ }^{1} \mathrm{H}$ NMR. The formic acid released was estimated from the ratio between the area of the peaks at $\delta 8.2 \mathrm{ppm}$ (formic acid) and $\delta 1.2 \mathrm{ppm}\left(\mathrm{CH}_{3}\right.$ from ethanol). The ethanol was added to the NMR tube in the beginning of the experiment.

\subsection{Preparation of dexOx hydrogels}

DexOx was dissolved to a final concentration of $28.6 \%$ $(\mathrm{w} / \mathrm{v})$, in $0.2 \mathrm{M}$ phosphate buffer $\mathrm{pH} 8.0$, for $16 \mathrm{~h}$, at $37^{\circ} \mathrm{C}$. Afterwards, $700 \mu \mathrm{L}$ of this solution was transferred to a 24 well tissue-culture plate and mixed with $300 \mu \mathrm{L}$ of AAD solution (dissolved in the same phosphate buffer) at different concentrations $(5,10$ and $20 \%$ in molar base taking into account the number of glucose residues in the original dextran) with the pipette tip. The crosslinking reaction was allowed to proceed overnight at room temperature.

\subsection{Dynamic swelling experiments}

DexOx gels after being prepared, and accurately weighted $\left(W_{\mathrm{i}}\right)$ were immersed in different $\mathrm{pH}$ buffers solutions (ca. $10 \mathrm{~mL}$ ) in sealed containers, at $37^{\circ} \mathrm{C}$. The buffer solutions used were $0.05 \mathrm{M} \mathrm{HCl} / \mathrm{KCl}$ for $\mathrm{pH} 1.0$; 0.2 M citrate-phosphate for $\mathrm{pH} 3.0-5.0 ; 0.01 \mathrm{M}$ PBS $\mathrm{pH} 7.4$ and $0.05 \mathrm{M}$ Tris $-\mathrm{HCl}$ for $\mathrm{pH}$ 9.0. At regular intervals, they were removed from the aqueous solution, blotted with filter paper to remove surface water, weighed $\left(W_{t}\right)$ and returned to the same container (the buffer solution was replaced at each measurement). The swelling index was calculated from the ratio between $W_{\mathrm{t}}$ and $W_{\mathrm{i}}$. In some cases, at different times, the hydrogels were lyophilized on a freeze-drier for $72 \mathrm{~h}$ and weighted $\left(W_{\mathrm{d}}\right)$. After $W_{\mathrm{d}}$ determination, either swelling ratio $\left(\mathrm{SR}=\left(W_{\mathrm{t}}-W_{\mathrm{d}}\right) / W_{\mathrm{d}}\right)$ or mass remaining $\left[\left(W_{\mathrm{d}} / W_{\mathrm{i}}\right) \times 100\right]$ was calculated. The gel-fraction of hydrogels was calculated from the ratio of the dry weight of hydrogel after swelling in citrate buffer $\mathrm{pH} 5.0$ for 2 days and the initial dry weight immediately after the polymerization reaction.

\subsection{Rheological analysis}

Hydrogels were equilibrated in $0.2 \mathrm{M}$ citrate buffer solution $\mathrm{pH} 5.0$ for 2 days, at room temperature, before being tested. Rheological experiments were carried out using the parallel plate geometry ( $20 \mathrm{~mm}$ diameter, steel) of a Haake Rheostress RS 1. Sandpaper was glued onto each plate surface in order to avoid slippage of samples. Equilibrium swelling conditions have been maintained during rheological measurements by adding water at $\mathrm{pH}$ 5.0 in the lower plate of the geometry until the entire free lateral surface of hydrogels was completely wetted by the liquid. Gap setting optimizations have been undertaken according to the procedure described elsewhere [21]. Hydrogels were subjected to stress sweep experiments (frequency of $1 \mathrm{~Hz}$ ) in order to optimize the applied stress used in the frequency-oscillation experiments. These last experiments were recorded over a frequency range from 0.1 to $10 \mathrm{~Hz}$. For the calculation of gelation period, both solutions were mixed on the bottom plate, and the upper plate was positioned at a gap of $1.1 \mathrm{~mm}$. This procedure took around $20 \mathrm{~s}$ and then the experiment was started at low frequency $(0.5 \mathrm{~Hz})$ and stress $(0.1 \mathrm{~Pa})$.

\subsection{Scanning electron microscopy (SEM) analysis}

Swollen dexOx gels were quickly frozen in a glass container using liquid nitrogen and freeze-dried for $72 \mathrm{~h}$. Fractured pieces of $0.6-0.9 \mathrm{~cm}$ in length, corresponding to half-diameter of each hydrogel, were mounted into an aluminum stud and gold coated by plasma vapor deposition. The surface and cross-section of hydrogels were recorded by a field emission scanning electron microscope (JEOL model JSM-5310), at 15.0-20.0 kV.

Analyses of the digitized images were performed using the public domain ImageJ $1.31 \mathrm{v}$ program (http://rsb.info. nih.gov/ij/), according to a methodology described by others $[14,22]$. Briefly, a threshold procedure was applied to the SEM images and the validity of the threshold level was confirmed by comparing the image before and after the procedure. In case of irregularities, they were corrected manually by comparison to the original photograph. After calibrating with a known scale, the pores were measured and labeled one by one. The diameter of a pore was obtained by averaging the major and the minor axes of the pore. 


\subsection{Mercury intrusion porosimetry (MIP)}

Mercury porosimetry (Micromeritics Poresizer 9320) was used to determine the bulk density and pore size distribution of freeze-dried hydrogels. The samples were degassed before analysis at a vacuum pressure below $50 \mu \mathrm{mHg}$. By knowing the volume intruded at $0.5-0.7$ psia (bulk volume: it includes solid sample, pores and interstices) and the material weight, it was possible to calculate the bulk density. High-pressure runs (from 25 up to 30,000 psia) were made with an equilibration time of $20 \mathrm{~s}$ and a maximum intrusion volume of $0.05 \mathrm{~mL} / \mathrm{g}$. The porograms (intruded volume versus pressure) obtained were converted into pore diameter distribution curves (cumulative and differential) according to the Washburn equation [23]:

$p d=-4 \gamma \cos \theta$

where $p$ is the pressure required to force mercury into a pore of entry diameter $d, \gamma$ is the surface tension (485 dyn $\mathrm{cm}^{-1}$ ), and $\theta$ is the contact angle between mercury and the sample $\left(130^{\circ}\right)$.

\section{Results and discussion}

\subsection{Characterization of dexOx}

Dextran was oxidized by sodium periodate at different extents. The periodate ion attacks one of the hydroxyl groups of the vicinal triol in dextran residues, between $\mathrm{C}_{3}-$ $\mathrm{C}_{4}$ or $\mathrm{C}_{2}-\mathrm{C}_{3}$ breaking the $\mathrm{C}-\mathrm{C}$ bond and yielding two aldehyde groups (Fig. 1). According to Ishak and Painter [24] the cleavage at the $\mathrm{C}_{3}-\mathrm{C}_{4}$ bond is favoured ca. 7.5-fold comparatively to the cleavage at $\mathrm{C}_{2}-\mathrm{C}_{3}$ bond. A second oxidation can occur since the aldehyde in position $\mathrm{C}_{3}$ has a vicinal hydroxyl group, which is susceptible to further attack, and this carbon is expelled under formic acid [24,25]. This is confirmed by the variation of $\mathrm{pH}$ during the timecourse reaction (Fig. 2). The results obtained showed that the reaction is very fast and after ca. $5 \mathrm{~min}$ the $\mathrm{pH}$ of the reaction mixture decreased from 5.5 to 2.5. After that time,

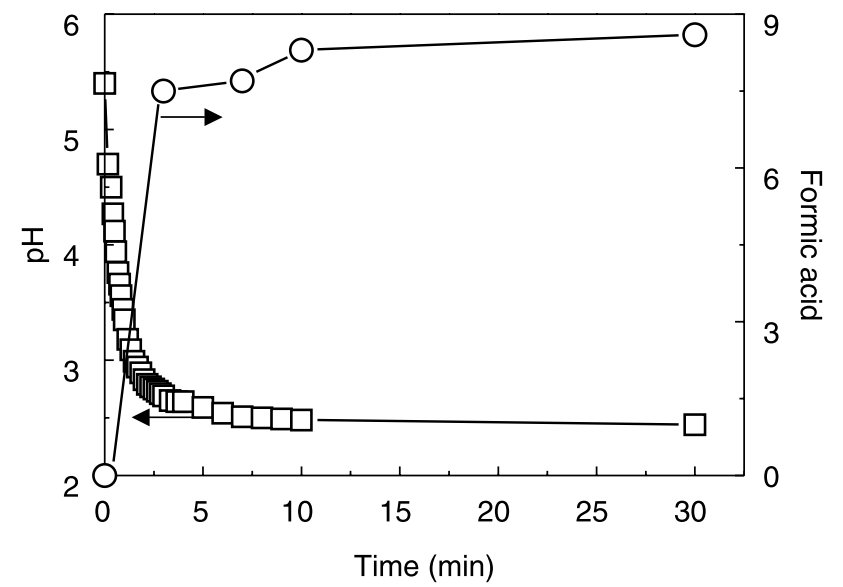

Fig. 2. Variation of $\mathrm{pH}(\square)$ and formic acid $(\bigcirc)$ during the oxidation reaction of dextran with sodium periodate. The formic acid release was followed by ${ }^{1} \mathrm{H}$ NMR, and calculated as the ratio between the area of the peaks at $\delta 8.2 \mathrm{ppm}$ (formic acid) and $\delta 1.2 \mathrm{ppm}\left(\mathrm{CH}_{3}\right.$ from ethanol). The ethanol was added to the NMR tube in the beginning of the experiment.

the $\mathrm{pH}$ of the reaction mixture stabilizes suggesting that no more formic acid is released. The release of formic acid was also followed by ${ }^{1} \mathrm{H}$ NMR (see below). According to Fig. 2, there is a burst release of formic acid during the first five minutes followed by a release at a slower rate.

The degree of oxidation of the dexOx samples was followed by tBC titration, as described elsewhere for other oxidized polysaccharides [11,19]. DexOx derivatives were allowed to react with an excess of $\mathrm{tBC}$ and the unreacted carbazates were quantified by their reaction with TNBS to form trinitrophenyl derivatives. The number of aldehydes in each sample was determined by subtracting the number of unreacted carbazates from the total number of added carbazates. Table 1 displays the theoretical degree of oxidation, which reflects the molar ratio of sodium periodate per initial glucose unit in dextran, and the obtained degree of oxidation calculated by the assay with tBC. As expected, the degree of oxidation of dexOx increased as the amount of added periodate increased. In addition, the degree of oxidation obtained experimentally for dexOx derivatives was lower than the theoretical values, and this may be

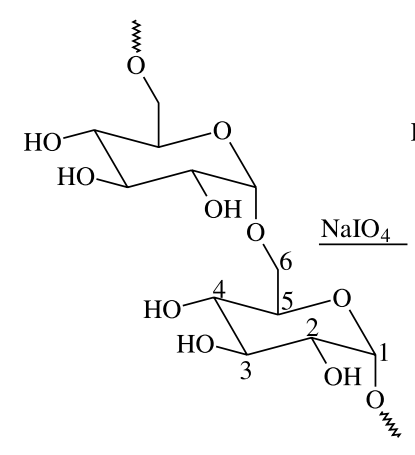

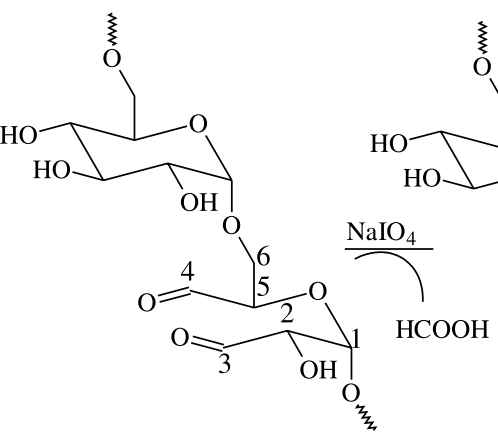

A

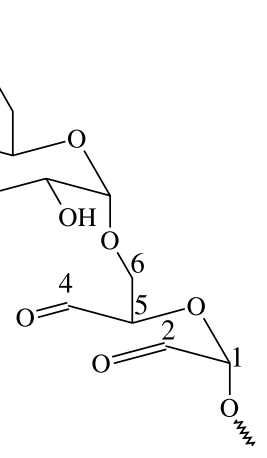

B

Fig. 1. Schematic representation of dextran oxidation by sodium periodate. (A) First oxidation with the concomitant formation of aldehyde groups at positions C3 and C4. (B) Second oxidation with the release of formic acid. 
Table 1

Degree of oxidation of several oxidized dextrans calculated by colorimetric and NMR analysis and number average of molecular weight $\left(M_{\mathrm{n}}\right)$ estimated by SEC

\begin{tabular}{|c|c|c|c|c|c|c|c|}
\hline \multirow[t]{2}{*}{ Entry } & \multirow[t]{2}{*}{$\begin{array}{l}\text { Isolated yield } \\
(\%)\end{array}$} & \multirow[t]{2}{*}{$\begin{array}{l}\text { Theoretical oxidation } \\
(\%)^{\mathrm{a}}\end{array}$} & \multicolumn{2}{|c|}{$\begin{array}{l}\text { Obtained degree of oxidation } \\
(\%)^{\mathrm{b}}\end{array}$} & \multirow[t]{2}{*}{$\begin{array}{l}\text { Doubly oxidized } \\
\text { units }(\%)^{\mathrm{c}}\end{array}$} & \multirow[t]{2}{*}{$M_{\mathrm{n}}(\mathrm{kDa})^{\mathrm{d}}$} & \multirow[t]{2}{*}{$M_{\mathrm{w}} / M_{\mathrm{n}}^{\mathrm{d}}$} \\
\hline & & & Colorimetric $^{\mathrm{e}}$ & ${ }^{1} \mathrm{H} \mathrm{NMR}^{\mathrm{f}}$ & & & \\
\hline 1 & 76.5 & 25 & $15.4 \pm 2.8$ & 14.3 & $7.1 \pm 0.1$ & 40.8 & 1.05 \\
\hline 2 & 65.0 & 10 & $8.7 \pm 1.8$ & 7.0 & $3.0 \pm 0.1$ & 52.2 & 1.05 \\
\hline 3 & 71.4 & 5 & $5.2 \pm 0.7$ & 4.2 & $1.3 \pm 0.1$ & 54.3 & 1.04 \\
\hline
\end{tabular}

a Calculated as the molar ratio of sodium periodate per initial glucose unit in dextran.

b DO defined as the number of oxidized residues per 100 glucose residues.

c Calculated by formic acid titration (average $\pm \mathrm{SD}, n=3$ ).

d Calculated by SEC analysis. The $M_{\mathrm{n}}$ and $M_{\mathrm{w}} / M_{\mathrm{n}}$ for the original dextran were 54.92 and $1.05 \mathrm{kDa}$, respectively.

e Calculated by the $\mathrm{tBC}$ assay (average $\pm \mathrm{SD}, n=3$ ).

${ }^{\mathrm{f}}$ Calculated by ${ }^{1} \mathrm{H}$ NMR taking into account the ratio between the integral of the peak at $\delta 7.3$ ppm and the integral of the anomeric proton at $\delta 4.9$ ppm.

explained by the double oxidation of the glucose units. The double-oxidation of the glucose units from dextran was quantified by the formic acid released during the oxidation reaction. According to Table 1, the percentage of doubly oxidized glucose increases with the sodium periodate concentration, but never represents the majority of the population of oxidized residues.

DexOx derivatives were then characterized by FTIR (Fig. 3). The FTIR spectra of dextran (A) and dexOx DO $15 \%$ (B) were very similar and it is hard to see any signal corresponding to the aldehyde functionalities. Previously, it has been reported that the band corresponding to the stretching of the carbonyl from an aldehyde group appears at $1730 \mathrm{~cm}^{-1}$ [11]. Such band was not observed in the present work for dexOx with DO upon 15\% (although a low intensity signal at $1730 \mathrm{~cm}^{-1}$ is observed in the FTIR spectrum of dexOx DO 50\% (results not shown)), and this may be due to the low degree of oxidation of dexOx and the formation of hemiacetals (see below).

DexOx derivatives were also characterized by NMR spectroscopy (Fig. 4). In the ${ }^{1} \mathrm{H}$ NMR spectrum of dextran

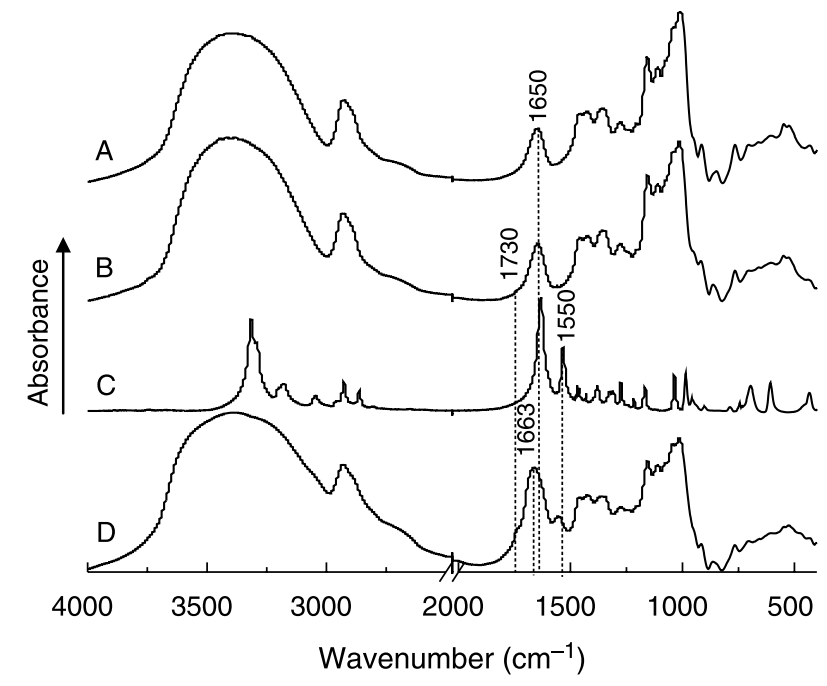

Fig. 3. FTIR spectra of dextran (A), dexOx DO 15\% (B), AAD (C), and gel of dexOx with $10 \%$ AAD (D).
(Fig. 4(A)), the peaks between $\delta 4.0$ and $3.4 \mathrm{ppm}$ are assigned to protons at positions $2,3,4,5$ and 6 , while the peak at $\delta 4.9 \mathrm{ppm}$ is attributed to the anomeric proton from the glucose unit. The spectrum also shows a small peak at $\delta$ $5.3 \mathrm{ppm}$ corresponding to the anomeric proton in dextran with $\alpha-1,3$ linkages $(\sim 2.5 \%$ of the total dextran [26]). To obtain information about the chemical changes during oxidation, the reaction was carried out in the NMR tube. For that purpose, dextran was dissolved in $\mathrm{D}_{2} \mathrm{O}$ and then an aqueous periodate solution was added to obtain a theoretical degree of oxidation of $100 \%$. Fig. 4(B) displays the ${ }^{1} \mathrm{H}$ NMR spectrum obtained after 10 min of reaction, showing several distinctive peaks in the region of $4.0-10.0 \mathrm{ppm}$ that were not present in the original dextran. The peak at $\delta$ $8.3 \mathrm{ppm}$ corresponds to the formic acid released during the oxidation reaction of dextran with sodium periodate. In addition, a peak with low intensity can be observed at $\delta$ 9.7 ppm (Fig. 4(B)), which is likely assigned to the protons of aldehyde groups. However this peak is not observed for reaction times above $30 \mathrm{~min}$ (data not shown), suggesting that during the oxidation reaction the aldehyde groups are converted into hemiacetals $[24,27]$. This is also confirmed by the appearance of several peaks in the ${ }^{1} \mathrm{H}$ NMR spectrum between $\delta 5.8$ and $\delta 4.2 \mathrm{ppm}$ (similar chemical shift range was reported for hemiacetals in the oxidized methyl glycopyranoside [27]) assigned to protons from different hemiacetal structures. The hemiacetals formed during the oxidation reaction are partially reverted by the addition of tBC. Fig. 4(C) and (D) shows the ${ }^{1} \mathrm{H}$ NMR spectrum of dexOx DO $15 \%$ before and after reaction with $\mathrm{tBC}$, respectively. In Fig. 4(D), there is a broad peak at $7.3 \mathrm{ppm}$ that is assigned to the proton attached to the carbon that was modified with $\mathrm{tBC}$. Conversely, the intensity of the peaks corresponding to protons in the hemiacetal structure (in the range of $\delta 5.6-5.0 \mathrm{ppm}$ ) decreased significantly.

The ${ }^{1} \mathrm{H}-{ }^{13} \mathrm{C}$ HMQC spectrum (bi-dimensional NMR experiment showing the correlations between protons and carbons; Fig. 5) further illustrate the formation of several hemiacetals, displaying several ${ }^{13} \mathrm{C}$ signals between 79 and $103 \mathrm{ppm}$ belonging to carbons in this cyclic structure. Intra- 


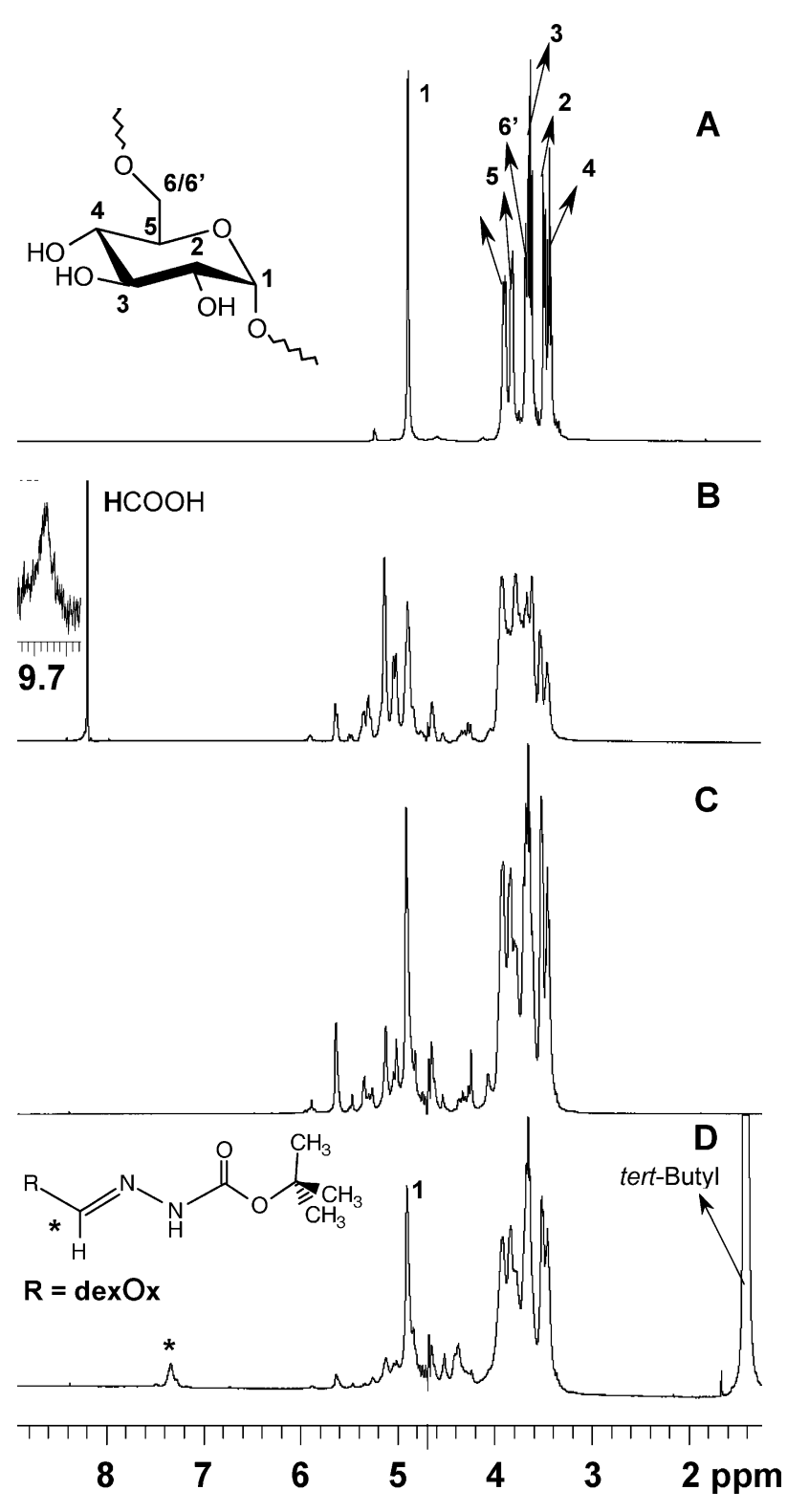

Fig. 4. ${ }^{1} \mathrm{H}$ NMR spectra of dextran (A), dexOx (100\% theoretical oxidation) after 10 min oxidation (B), dexOx DO 15\% (C) and dexOx DO 15\% reacted with $\mathrm{tBC}(\mathrm{D})$.

residue as well inter-residue hemiacetals may be expected in the single and double oxidation of the glucose, respectively, according to previous reports [24,27]. According to the ${ }^{1} \mathrm{H}-{ }^{13} \mathrm{C}$ HMQC spectrum (Fig. 5(B)) the ${ }^{1} \mathrm{H}$ peak at $7.3 \mathrm{ppm}$, (Fig. 4(D)) is correlated with a ${ }^{13} \mathrm{C}$ peak at $\delta$ $146.5 \mathrm{ppm}$, and thus the carbon chemical shift was moved to low field (as compared to carbons belonging to the hemiacetal structure) due to the presence of $\pi$ electrons belonging to the carbazone functionality. NMR simulation studies (using ACD software from Advanced Chemical Development, Inc.), indicate that this proton is located at position 4 from a previous aldehyde group. In case of reaction of $\mathrm{tBC}$ with the hemiacetal at position $\mathrm{C}_{2}$ and $\mathrm{C}_{3}$, a peak in the same chemical shift range would be expected;

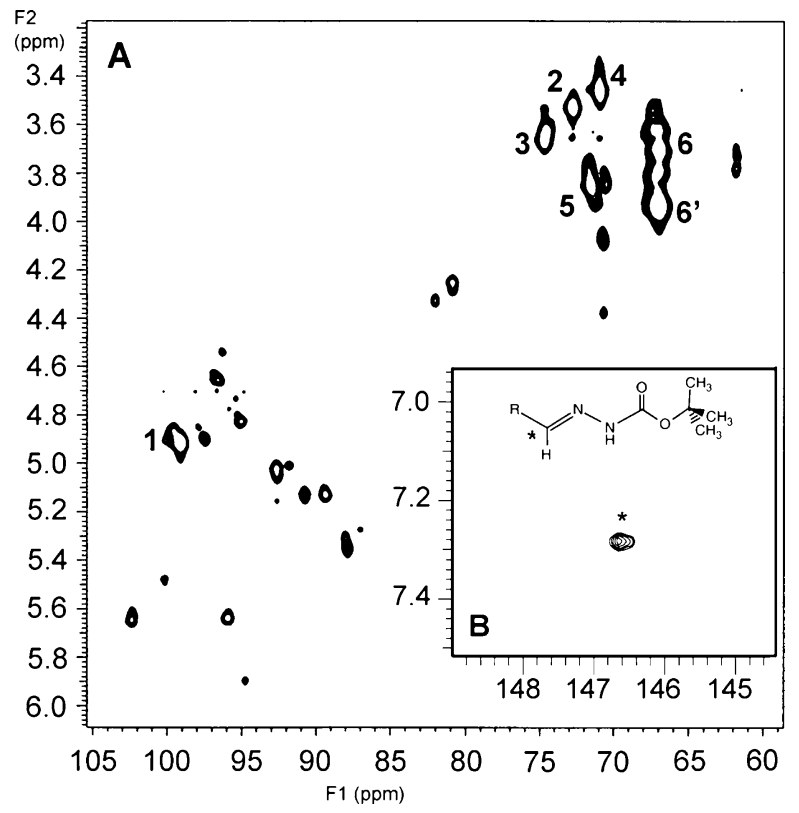

Fig. 5. ${ }^{1} \mathrm{H}-{ }^{13} \mathrm{C}$ HMQC spectrum of dexOx DO $15 \%$ (A). Detail of a ${ }^{1} \mathrm{H}-{ }^{13} \mathrm{C}$ HMQC spectrum of dexOx DO $15 \%$ coupled with $\mathrm{ABC}$ showing the new correlation from the newly formed carbazone (B).

however, no peak is observed. This indicates that the hemiacetal involving the carbon at position 2 or 3 is not reverted by the $\mathrm{tBC}$.

SEC analyses (Table 1) showed that the $M_{\mathrm{n}}$ of dexOx decreased as compared to the original dextran. This is likely due to the cleavage of the dextran backbone with the oxidation [28]. Similar results have been reported in the oxidation of hyaluronic acid [19] and polyguluronate [13].

\subsection{Preparation and characterization of dexOx hydrogels}

Aqueous solutions of dexOx DO $15 \%$ were crosslinked with various concentrations of adipic dihydrazide $(5,10$ and $20 \%$ in molar ratio of AAD to glucose units on dextran) at room temperature. The hydrazide group in AAD reacts with the aldehyde groups in dexOx to form hydrazone bonds, which can be hydrolyzed yielding again dexOx and $\mathrm{AAD}$ (Fig. 6(A)). The hydrogels were soaked with $0.2 \mathrm{M}$ citratephosphate $\mathrm{pH} 5.0$ for $48 \mathrm{~h}$ to release the unreacted AAD and then lyophilised (at this $\mathrm{pH}$ the gels have long dissolution time, see below). As shown in Table 2, the gel fractions of dexOx hydrogels were ca. $100 \%$. Therefore, most of the dexOx derivatives formed covalent linkages with AAD. This was further confirmed by FTIR spectroscopy. According to Fig. 3(C), the FTIR spectrum of the hydrogel shows the appearance of a band at $1663 \mathrm{~cm}^{-1}$ that corresponds to the stretching vibration of the carbonyl group in the hydrazone bonds as well the stretching of $\mathrm{C}=\mathrm{N}$ [11]. Furthermore, the band at $1550 \mathrm{~cm}^{-1}$ likely corresponds to the bending of $\mathrm{NH}$ [29].

Gelation of the reaction mixtures was observed by dynamic rheological measurements at a fixed frequency and 

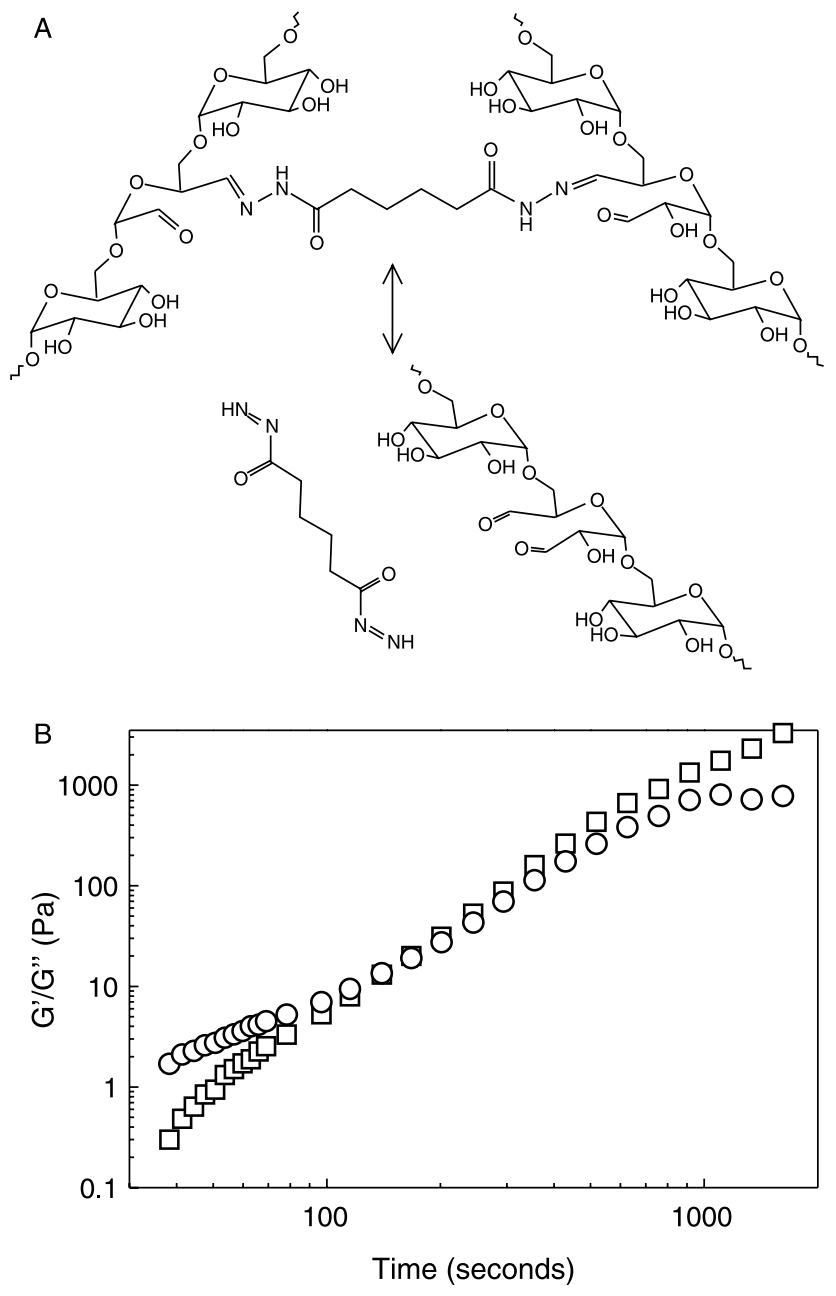

Fig. 6. (A) Scheme for the crosslinking of dexOx with $\mathrm{AAD}$ and the corresponding degradation products of the network. (B) $G^{\prime}(\square)$ and $G^{\prime \prime}$ (O) as a function of crosslinking time of dexOx DO $15 \%$ with $5 \%$ AAD.

Table 2

Swelling and mechanical properties of dexOx hydrogels DO $15 \%$ crosslinked with various concentrations of AAD

\begin{tabular}{lccc}
\hline Property & \multicolumn{3}{l}{ AAD content } \\
\cline { 2 - 4 } & $5 \%$ & $10 \%$ & $20 \%$ \\
\hline Gel fraction $(\%)^{\mathrm{a}}$ & 100 & 100 & 100 \\
$\mathrm{G}^{\prime}(\mathrm{kPa})^{\mathrm{b}}$ & 7.0 & 20.7 & 32.0 \\
${\mathrm{Gelation} \mathrm{period}(\mathrm{s})^{\mathrm{c}}}_{\mathrm{SR} 37^{\circ} \mathrm{C}^{\mathrm{a}, \mathrm{d}}}$ & 230 & 150 & 117 \\
$\bar{M}_{\mathrm{c}}\left(\mathrm{g} \mathrm{mol}^{-1}\right)^{\mathrm{e}}$ & 8.7 & 4.3 & 3.8 \\
$\rho\left(\times 10^{-3} \mathrm{~mol} \mathrm{~cm}^{-3}\right)^{\mathrm{f}}$ & 716 & 201 & 127 \\
\hline
\end{tabular}

${ }^{a}$ The values were determined after hydrogels being immersed on citratephosphate $\mathrm{pH} 5.0$ buffer for 2 days.

${ }^{\mathrm{b}} G^{\prime}$ was calculated through a frequency sweep measurement.

c The gelation period was considered when $G^{\prime}=G^{\prime \prime}$.

${ }^{\mathrm{d}}$ The equilibrium swelling ratio (SRE) was attained when $W_{\mathrm{s}}$ (Section 2) stabilization was observed (less than $2 \%$ change in $48 \mathrm{~h}$ ).

${ }^{\mathrm{e}}$ Molecular weight between crosslinks.

${ }^{\mathrm{f}}$ Crosslinking density. amplitude. Fig. 6(B) represents the typical behavior of $G^{\prime}$ (which is a measure of the energy stored and recovered per cycle of oscillatory deformation and therefore represents the elastic behaviour of the material [30]) and $G^{\prime \prime}$ (measure of energy dissipation per cycle of sinusoidal deformation and represents the viscous behavior of the system [30]) during the gelation period. On the first stage (pregelation period) the values of $G^{\prime \prime}$ were higher than $G^{\prime}$ showing essentially a liquid behavior of the system. After some time, the $G^{\prime}$ increases suddenly due to the formation of elastic intermolecular crosslinks. The crossover point, where $G^{\prime}$ is equal to $G^{\prime \prime}$, provides an accurate measure for the gelation threshold, marking the transition from predominantly viscous to elastic properties [30,31]. The results shown in Table 2, suggest that gelation of dexOx with AAD is very rapid with time ranges between 2 and 4 min for all the reaction mixtures tested. These results can be compared favourably to those obtained when the crosslinking reaction of other polysaccharides is carried out by using free radical polymerisation with the use of chemical initiators. For example, Vervoort et al. [30] reported gelation times for inulin acrylate derivatives in the order of $16 \mathrm{~min}$, although faster gelation times could be reached by increasing abruptly the concentrations of the free radical initiators. In addition, Smedt et al. [31] reported gelation times of $5 \mathrm{~min}$ for the gelation of dextran-acrylate derivatives.

The time period to complete the gelation process is quite large on dexOx. The time needed to achieve a constant $G^{\prime}$ was always above $3 \mathrm{~h}$, showing that the curing process was long. The hydrogels after being prepared were immersed on citrate-phosphate buffer $\mathrm{pH} 5.0$ for 2 days and then characterized by rheology. According to Table 2, the $G^{\prime}$ increases with the concentration of AAD. The values found are higher than the ones obtained for other polysaccharidebased hydrogels such as poly(aldehyde guluronate) $\left(G^{\prime}\right.$ between 1.4 and $6.1 \mathrm{kPa}$ ) [13] and dextran-methacrylate hydrogels $\left(G^{\prime}\right.$ between 0.1 and $\left.10 \mathrm{kPa}\right)$ [31] with different degrees of substitution, albeit lower than the values observed for inulin-methacrylate hydrogels $\left(G^{\prime}\right.$ between 5 and $60 \mathrm{kPa}$ ) [30]. The $G^{\prime}$ can be converted into crosslinking density $\left(\rho_{x}\right)$ by the use of phantom network theory [32] through Eqs. (2) and (3):

$$
\begin{aligned}
G^{\prime} & =\frac{\rho R T}{\bar{M}_{\mathrm{c}} \nu_{2, \mathrm{~s}}^{1 / 3}}\left(1-\frac{2 \bar{M}_{\mathrm{c}}}{M_{\mathrm{n}}}\right) \\
\rho_{x} & =\frac{1}{\bar{\nu} \bar{M}_{\mathrm{c}}}
\end{aligned}
$$

where $\rho$ is the polymer density $\left(0.96 \mathrm{gm} \mathrm{L}^{-1}\right), R$ is the gas constant $\left(8.314 \mathrm{~J} \mathrm{~K}^{-1} \mathrm{~mol}^{-1}\right), T$ is the temperature $(298 \mathrm{~K})$, $\bar{M}_{\mathrm{c}}$ is the average molecular weight between crosslinks, $\bar{M}_{\mathrm{n}}$ is the number average molecular weight of dextran $(39,940 \mathrm{Da}), \nu_{2, \mathrm{~s}}$ is the polymer fraction at equilibrium swelling (Table 2) and $\bar{\nu}$ is the partial specific volume of dextran $\left(0.62 \mathrm{~cm}^{3} \mathrm{~g}^{-1}\right)$ [33]. As expected, the crosslinking 
density increases with the increasing concentration of AAD in the hydrogel formulation. The values obtained are lower than the ones obtained for oxidized polyguluronate [13] and oxidized chondroitin gelatin hydrogels [34] but in these cases the degree of oxidation of the initial polymer was much higher $(66.5 \%$ for polyguluronate and $40-60 \%$ for chondroitin gelatin hydrogels).

The swelling of a hydrogel is of utmost importance in biomedical and pharmaceutical applications since it influences the solute diffusion coefficient through the network, the surface properties and surface mobility and the mechanical properties [35]. The swelling pattern of dexOx DO 15\% hydrogels crosslinked with $10 \%$ AAD was characterized at different $\mathrm{pH}$ values. At $\mathrm{pH} 1.0$, the hydrogels undergo degradation in less than $2 \mathrm{~h}$ due to hydrolysis of $\alpha-1,6$ glucosidic linkages [33]. At $\mathrm{pH} 9.0$, the hydrogels are completely degraded in ca. $24 \mathrm{~h}$, due to hydrolysis of the hydrazone bonds. However, the degradation at $\mathrm{pH}$ 's 3 and 5 was much slower, being the networks completely solubilized after 40 and 50 days, respectively (results not shown). At pH 5.0, the swelling ratio (SR) of hydrogels decreases with the increase of AAD content (Table 2). The swelling values at this $\mathrm{pH}$ are close to the ones observed in dextran-acrylate hydrogels (SR between 8.6 and 3.7) [2], but lower than the values observed for poly(aldehyde guluronate) hydrogels (SR between 12.6 and 15.3) [13] and networks obtained from oxidized hyaluronic acid (SR between 25 and 65) [19].

\subsection{Degradation of dexOx hydrogels at physiologic $p H$}

Fig. 7 shows the swelling index and the mass loss profile of dexOx DO $15 \%$ hydrogels with different crosslinking densities as a function of time, when incubated in $10 \mathrm{mM}$ PBS at $\mathrm{pH}$ 7.4. For dexOx hydrogel crosslinked with 5\% $\mathrm{AAD}$, there is a slight increase in the swelling index for the first $24 \mathrm{~h}$ followed by a decrease during 9 days upon complete dissolution. During this time, the hydrogel undergoes hydrolysis and the crosslink junctions are degraded almost linearly with time. Since few junction points are present in the network, this has a direct effect in the mass loss. For high crosslinked hydrogels (AAD content of 10 and 20\%), the swelling index increases during the first 12-14 days and then decreases until the networks are completely solubilized, in 15-23 days. The increase of swelling during time is a consequence of the hydrolysis of the crosslinks (hydrazone bonds) in the hydrogel network and not dextran, which is stable at this $\mathrm{pH}$ [33]. When crosslinks in the hydrogel, are hydrolyzed, the network swells and imbibes more water. Hydrogels with higher AAD content and therefore with higher number of intermolecular crosslinks (as demonstrated by rheological analysis), tend to hydrolyze slower than the ones with lower AAD content. According to the mass loss studies, the degradation pattern found in hydrogels crosslinked with 10 and $20 \%$ AAD is
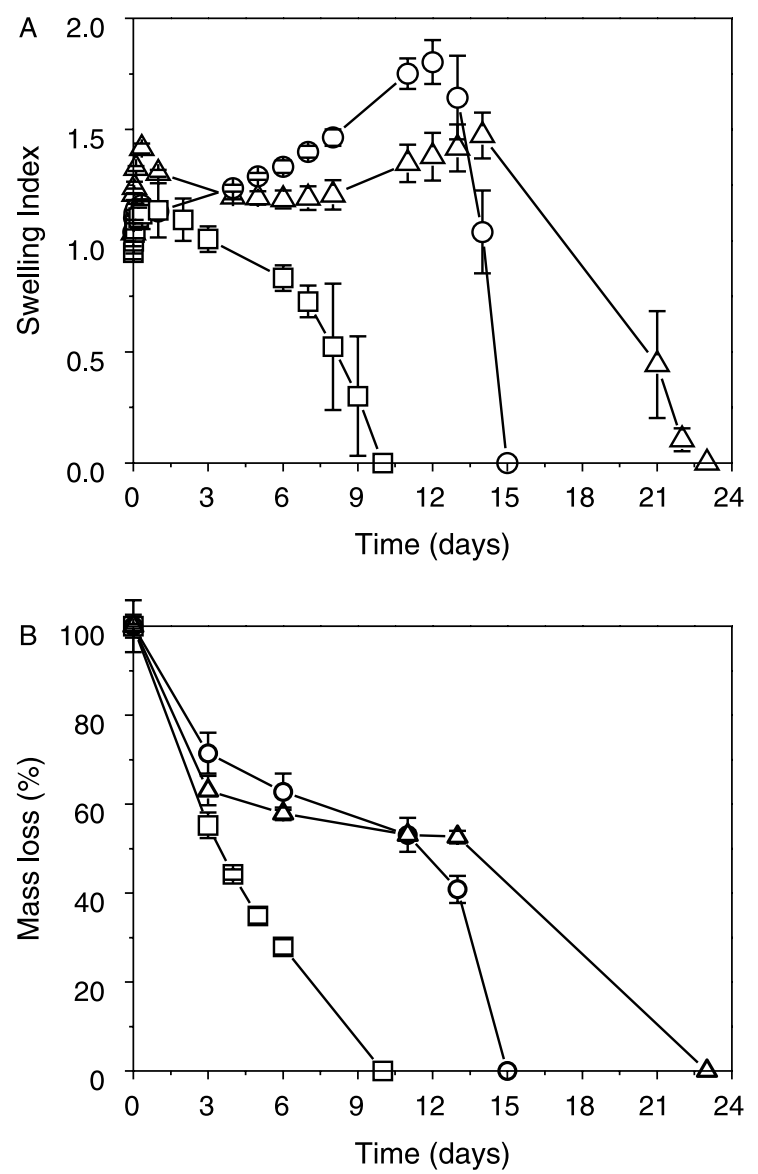

Fig. 7. Swelling index (A) and mass loss (B) studies of dexOx DO $15 \%$ hydrogels with different crosslinking densities: $5 \%(\square), 10 \%(\bigcirc)$ and $20 \%$ $(\diamond)$ of AAD, in PBS solution at $37^{\circ} \mathrm{C}$.

different from the one observed in hydrogels crosslinked with 5\% AAD. In the former, there is an initial decrease in the mass loss (ca. 25-35\%) for the first 3 days, likely due to the hydrolysis of the hydrogel regions with low crosslinking density. This phase was followed by a slight decrease in the mass during the next 10 days, corresponding likely to the degradation of high crosslinked regions, and a final dissolution phase.

It is important to study the variation of pore size during the degradation of the network since it affects the swelling of the hydrogel, the diffusion of macromolecules, and the delivery of cells when these hydrogel networks are used for cell encapsulation [5,15,17]. Consequently, the pore size range in dexOx hydrogels crosslinked with $10 \%$ of $\mathrm{AAD}$ was evaluated by mercury intrusion porosimetry (MIP), during their degradation at physiologic $\mathrm{pH}$. It should be noted that few studies have used this technique to evaluate pore size in hydrogels $[14,16,36]$. No references, related to the use of this technique to estimate hydrogel pore sizes during their degradation, were found in the literature. MIP measures the intruded volume of mercury in the sample at specific pressures. Then it converts the pressure into pore size according to the mathematical model of Washburn [23]. 
The applied pressure is inversely proportional to the diameter of pores intruded by mercury according to Eq. (1). MIP assumes that all pores are cylindrical and the diameter will correspond to the maximum strangling of the pore. According to Table 3, the total intruded volume of mercury increases as a function of degradation time of the network. This is due to the low number of crosslinks and thus more empty spaces in the degraded networks. In contrast, the bulk density (the volume includes solid sample, pores and interstices and possibly some compression contribution [36]) decreased as a function of degradation time. This is due to the loss of hydrogel mass during the degradation process and the concomitant increase of volume in the interior of the network. Finally, as the degradation process proceeds, the mode of the peak corresponding to the pores present in the network is shifted for higher pore sizes. It is evident from Fig. 8, that the degradation process implies larger intruded mercury volumes and broader pore size distributions, which is also expressed in Table 3, where we can observe that the pore size range of dexOx hydrogels increases from $0.08-0.32$ to $0.15-10.0 \mu \mathrm{m}$ after 11 days in PBS incubation.

The results obtained by MIP were further confirmed by SEM. SEM is very often described in the literature to study morphological details of hydrogels $[14,19,22,36]$. In addition, by using image analysis, it is possible to obtain information about pore size distribution; however, such information is restricted to the location where the analyses are performed. From Fig. 9 and Table 3 it is clear that pore size increased during the degradation of the network, from ca. 1.5 upon to $6.3 \mu \mathrm{m}$ after 11 days of degradation in PBS solution. It should be noted the close agreement between the pore size range obtained by MIP and SEM during the degradation of the networks, clearly indicating that the hydrogels are macroporous, and the compressibility effect can be considered negligible. This is clearly distinct from the behavior reported for hyperporous polyurethane-based gels where a compressibility effect was observed during MIP analysis [36].

Table 3

Characteristics of hydrogels obtained from the crosslinking of dexOx DO $15 \%$ with $10 \%$ AAD at different degradation times in $10 \mathrm{mM}$ PBS pH 7.4, as measured by SEM and MIP

\begin{tabular}{lllll}
\hline Time (days) & MIP & & SEM \\
\cline { 2 - 4 } & $\begin{array}{l}\mathrm{TIV}^{\mathrm{a}} \\
(\mathrm{mL} / \mathrm{g})\end{array}$ & $\rho_{\mathrm{b}}{ }^{\mathrm{b}}(\mathrm{g} / \mathrm{mL})$ & $\begin{array}{l}\text { Diameter } \\
\text { range }^{\mathrm{c}}(\mu \mathrm{m})\end{array}$ & $\begin{array}{l}\mathrm{l}_{\text {Diameter }} \\
\text { range }^{\mathrm{d}}(\mu \mathrm{m})\end{array}$ \\
\hline 0 & 1.88 & 0.3205 & $0.32-0.08$ & $1.50-0.29$ \\
3 & 2.62 & 0.2914 & $1.69-0.10$ & $2.06-0.38$ \\
6 & 3.65 & 0.2274 & $4.01-0.06$ & $5.54-0.63$ \\
11 & 3.77 & 0.1847 & $10.0-0.15$ & $6.33-1.70$ \\
\hline
\end{tabular}

${ }^{a}$ Total intrusion volume.

b Bulk density obtained by MIP at pressures around 0.5 psia.

c Pore size ranges considered for intruded values above the $0.6 \mathrm{~mL} / \mathrm{g}$ in the Fig. 9.

d Obtained from SEM images (cross-section) at magnification of $1000 \times$.

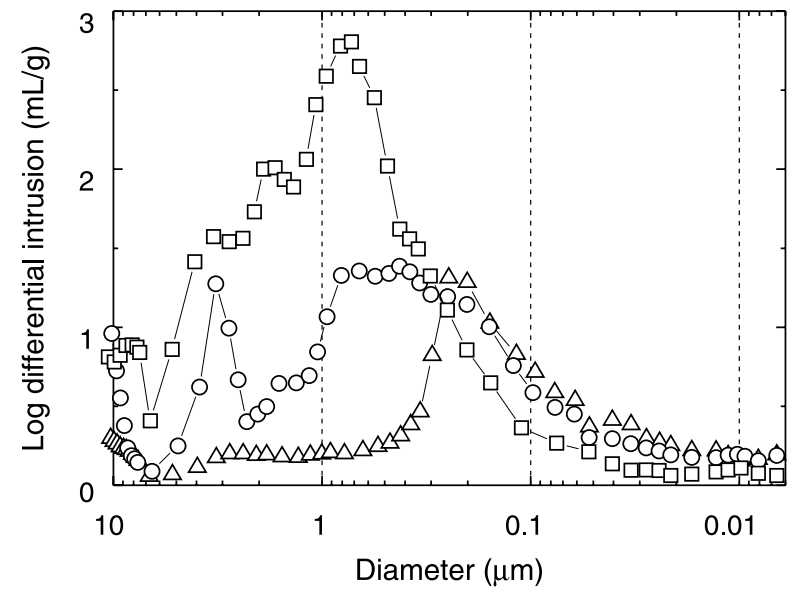

Fig. 8. Plot of pore size distribution (log differential intrusion) versus pore diameter of dexOx DO $15 \%$ hydrogels with $10 \%$ AAD before $(\diamond)$ and after the degradation process for $6(\bigcirc)$ and $11(\square)$ days in $10 \mathrm{mM}$ PBS buffer at $\mathrm{pH} 7.4$.

The degradation and macroporous structure of dexOx hydrogels (before and during degradation) make these networks attractive for the design of injectable protein/peptide delivery systems and scaffolds for tissue engineering. It has been reported that dense regions in non-degradable dextran-based hydrogels (ascribed to the existence of mesopores) reduce the release efficiency of proteins [15]. Therefore the macroporous structure and degradability of dexOx hydrogels may solve this problem. In addition, the increase of pore size during the degradation of dexOx hydrogels may contribute for the ingrowth of cells at the implant site and thus contributing for a better integration of the network with the surrounding tissue $[3,37]$.

\section{Conclusions}

This work reports the synthesis and characterization of new injectable and degradable dextran-based hydrogels. This formulation does not require the use of any chemical initiator and thus may be an alternative to other formulations for the preparation of hydrogels. In a first step, dextran was oxidized by sodium periodate, and then crosslinked by adipic acid dihydrazide forming a hydrogel in 2-4 min. The fast gelation of this mixture compares favorably to other formulations where free radical initiators were used. The results obtained by NMR suggest that the oxidation of dextran favored the formation of hemiacetals, which are reverted by the addition of tert-butyl carbazate or AAD, and a significant part (between 25 and $46 \%$ ) of the oxidized glucose units were doubly oxidized. The hydrogels prepared with different crosslinking densities presented acceptable mechanical properties with $G^{\prime}$ values ranging from 7 up to $32 \mathrm{kPa}$. Finally, the hydrogels were degraded at physiologic conditions being the hydrolysis mechanism slightly different depending on the crosslinking density of the network. 

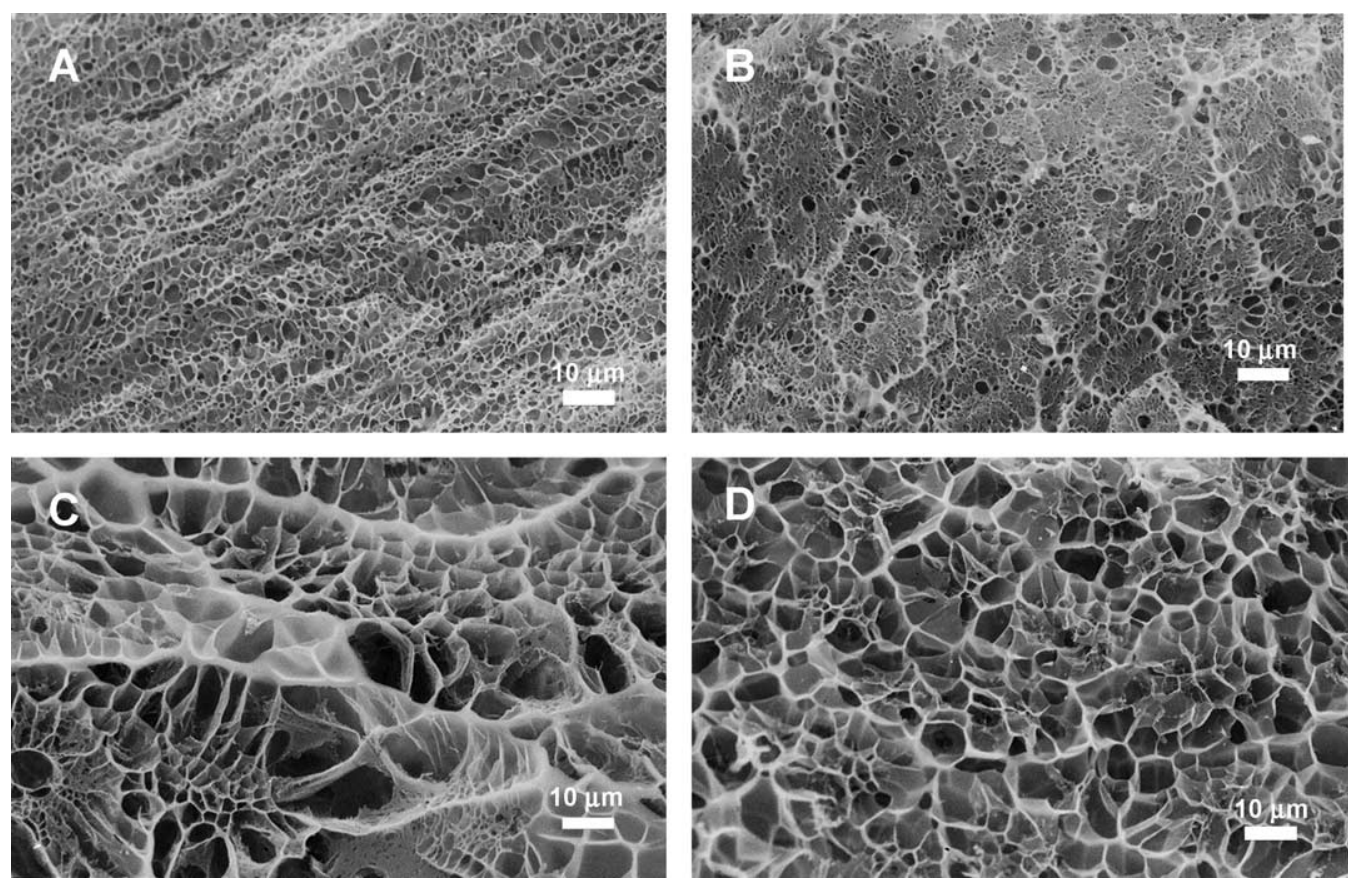

Fig. 9. SEM images from the cross-section of dexOx DO 15\% hydrogels with 10\% AAD before (A) and after immersion on PBS buffer for 3 days (B), 6 days (C) and 11 days (D).

The results obtained by SEM and MIP showed that pore size increases as a function of degradation time due to the hydrolysis of hydrazone bonds. In future work we envision using these hydrogels to design drug delivery systems as well as scaffolds for tissue engineering purposes.

\section{Acknowledgements}

The authors would like to thank Dr Margarida Figueiredo for the use of mercury porosimetry, Dr Graça Rasteiro for the use of rheological techniques, Dr Dmitry Evtiouguine for the use of the SEC, Fundação para a Ciência e Tecnologia for the financial support (SFRH/BPD/14502/2003) to LF and also to the ESPD group at Genentech, Inc. for allowing confirmation of some results.

\section{References}

[1] Ferreira L, Carvalho R, Gil MH, Dordick JS. Biomacromolecules 2002;3:333.

[2] Ferreira L, Gil MH, Dordick JS. Biomaterials 2002;23:3957.

[3] Ferreira L, Rafael A, Lamghari M, Barbosa MA, Gil MH, Cabrita AMS, et al. J Biomed Mater Res 2004;68A:584.

[4] Lee KY, Mooney DJ. Chem Rev 2001;101(7):1869.

[5] Peppas NA, Bures P, Leobandung W, Ichikawa H. Eur J Pharma Biopharm 2000;50:27.

[6] Wong WH, Mooney DJ. In: Atala A, Mooney DJ, editors. Synthetic biodegradable polymer scaffolds. Boston: Birkhäuser; 1997. p. 49-82.

[7] Mehvar R. J Controlled Release 2000;69:1.
[8] Nguyen KT, West JL. Biomaterials 2002;23:4307.

[9] Liu VA, Bhatia SN. Biomed Microdevices 2002;4(4):257.

[10] Williams CG, Malik AN, Kim TK, Manson PN, Elisseeff JH. Biomaterials 2005;26:1211

[11] Bouhadir KH, Hausman DS, Mooney DJ. Polymer 1999;40:3575.

[12] Lee KY, Alsberg E, Mooney DJ. J Biomed Mater Res 2001;56:228.

[13] Lee KY, Bouhadir KM, Mooney DJ. Macromolecules 2000;33:97.

[14] Kim S-H, Chu CC. J Biomed Mater Res, Appl Biomater 2000;53:258.

[15] Meyvis T, De Smedt S, Stubbe B, Hennink WE, Demeester J. Pharm Res 2001;18(11):1593.

[16] Dziubla TD, Torjman MC, Joseph JI, Murphy-Tatum M, Lowman AM. Biomaterials 2001;22:2893.

[17] Jen AC, Wake MC, Mikos AG. Biotechnol Bioeng 1996;50:357.

[18] Schneerson R, Barrera O, Sutton A, Robbis JB. J Exp Med 1980;152: 361.

[19] Jia X, Burdick JA, Kobler J, Clifton RJ, Rosowski JJ, Zeitels SM, et al. Macromolecules 2004;37:3239.

[20] Edwards-Lévy F, Andry MC, Lévy MC. Int J Pharm 1993;96:85.

[21] Meyvis TKL, De Smedt SC, Demeester J, Hennin WE. J Rheol 1999; 43:933.

[22] Behravesh E, Jo S, Zygourakis K, Mikos AG. Biomacromolecules 2002;3:374

[23] Washburn EW. Proc Natl Acad Sci 1921;7:115.

[24] Ishak MF, Painter TJ. Carbohyd Res 1978;64:189.

[25] Tiziani S, Sussich F, Cesáro A. Carbohyd Res 2003;338:1083.

[26] Van Dijk-Wolthuis WNE, Franssen O, Talsma H, Van Steenbergen MJ, Kettenes-Van den Bosch JJ, Hennink WE. Macromolecules 1995;28:6317.

[27] Yu RJ, Bishop CT. Can J Chem 1967;45:2195.

[28] Domb AJ, Linden G, Polacheck I, Benita S. J Polym Sci, Part A: Polym Chem 1996;34:1229.

[29] Pavia DL, Lampma GM, Kriz GS. Introduction to spectroscopy. 2nd ed. London: Saunders College Publishing; 1996 [Appendix I].

[30] Vervoort L, Vinckier I, Moldenaers P, Van den Mooter G, Augustijns P, Kinget R. J Pharm Sci 1999;88:209. 
[31] Smedt SCD, Lauwers A, Demeester J, Van Steenbergen MJ, Hennink WE, Roefs SPFM. Macromolecules 1995;28:5082.

[32] Bell CL, Peppas NA. Adv Polym Sci 1995;122:125.

[33] Van Dijk-Wolthuis WNE, Hoogeboom JAM, Van Steenbergen MJ, Tsang SKY, Hennink WE. Macromolecules 1997;30:4639.

[34] Dawlee S, Sugandhi A, Balakrishnan B, Labarre D, Jayakrishnan A. Biomacromolecules 2005;6:2040.
[35] Peppas NA. Hydrogels. In: Ratner BD, Hoffman AS, Schoen FJ, Lemons JE, editors. Biomaterials science: An introduction to materials in medicine. London: Academic Press; 1996. p. 60.

[36] Pirard R, Rigacci A, Maréchal JC, Quenard D, Chevalier B, Achard P, et al. Polymer 2003;44:4881.

[37] Ferreira L, Gil MH, Cabrita AMS, Dordick JS. Biomaterials 2005;26: 4707. 